\title{
OPEN The Electro-Optical Performance of Silver Nanowire Networks
}

Received: 5 March 2019

Accepted: 23 July 2019

Published online: 09 August 2019

\author{
Hugh G. Manning $\mathbb{D}^{1,4}$, Claudia Gomes da Rocha ${ }^{2}$, Colin O' Callaghan ${ }^{3,4}$, Mauro S. Ferreira ${ }^{3,4}$ \& \\ John J. Boland ${ }^{1,4}$
}

Networks of metallic nanowires have the potential to meet the needs of next-generation device technologies that require flexible transparent conductors. At present, there does not exist a first principles model capable of predicting the electro-optical performance of a nanowire network. Here we combine an electrical model derived from fundamental material properties and electrical equations with an optical model based on Mie theory scattering of light by small particles. This approach enables the generation of analogues for any nanowire network and then accurately predicts, without the use of fitting factors, the optical transmittance and sheet resistance of the transparent electrode. Predictions are validated using experimental data from the literature of networks comprised of a wide range of aspect ratios (nanowire length/diameter). The separation of the contributions of the material resistance and the junction resistance allows the effectiveness of post-deposition processing methods to be evaluated and provides a benchmark for the minimum attainable sheet resistance. The predictive power of this model enables a material-by-design approach, whereby suitable systems can be prescribed for targeted technology applications.

Modern photovoltaics, light-emitting devices, touch screens and thin-film transparent heaters all rely on a transparent conductor (TC) layer for operation. The most commonly used material for TCs has been ITO (tin-doped indium oxide), however, the brittle nature of the ITO film makes it incompatible with flexible device platforms ${ }^{1}$. Moreover, the scarcity of indium and the high cost of the ITO film deposition has motivated the search for alternative materials, which now includes conductive polymers ${ }^{2}$, carbon nanotubes ${ }^{3}$, graphene ${ }^{4}$, metal mesh $^{5}$, crackle networks ${ }^{6}$ and networks composed of metallic nanowires such as $\mathrm{Ag}, \mathrm{Au}$ and $\mathrm{Cu}^{7-9}$. Nanowire networks (NWNs) have demonstrated excellent optical, electrical and mechanical performances through low-temperature high-throughput fabrication techniques such as spray deposition ${ }^{10}$, Mayer rod coating ${ }^{11}$, and roll-to-roll slot die printing ${ }^{12}$. In particular, Ag NWNs can not only match the electro-optical performance of ITO, but can also fulfil the demands for the emerging flexible electronics market ${ }^{13}$. Next-generation devices such as flexible solar cells $^{14}$, touch screens ${ }^{15}$, displays ${ }^{16}$, thin-film heaters ${ }^{17}$, wearables ${ }^{18,19}$ and anti-static coatings ${ }^{20}$ require flexible $^{2}$ electro-optical components. Each TC film requires a high optical transmittance value $(T>90 \%)$ whereas the electrical requirements of the sheet resistance $\left(R_{\mathrm{s}}\right)$ is application-specific ${ }^{4}$. Figure 1 compares the current performance requirements of several proposed TC materials to ITO on a $T-R_{s}$ curve against the back drop of the requirements for a range of technologies. It is clear that Ag NWNs can fulfil the required optical and electrical performances for many technologies by tuning the $R_{\mathrm{s}}$ value. In the case of ITO, the optical and electrical properties are modulated by changing the film thickness ${ }^{21}$. For Ag NWNs, performance depends on the wire density $\left(n_{\mathrm{w}}\right)$, which, for the idealised case of a network made with wires of the same length and diameter is related to the junction density $\left(n_{\mathrm{j}}\right)$, the nanowire length $(L)$ and a contact probability $(P=0.2027)$ through Eq. 1 below $^{22}$

$$
n_{w}=\sqrt{\frac{2 n_{j}}{P \pi L^{2}}}
$$

The network $R_{\mathrm{s}}$ is ultimately controlled by the junction resistance $\left(R_{\mathrm{jxn}}\right)$ associated with overlapping Ag nanowires (NWs) in the network. $R_{\mathrm{jxn}}$ is a consequence of an electrically insulating few $\mathrm{nm}$ thick polyvinolpyrollidone (PVP) layer that forms a metal-insulator-metal configuration where ever NWs overlap to form a junction ${ }^{23}$.

\footnotetext{
${ }^{1}$ School of Chemistry, Trinity College Dublin, Dublin 2, Ireland. ${ }^{2}$ Department of Physics and Astronomy, University of Calgary, 2500 University Drive NW Calgary, Alberta, T2N 1N4, Canada. ${ }^{3}$ School of Physics, Trinity College Dublin, Dublin 2, Ireland. ${ }^{4}$ Centre for Research on Adaptive Nanostructures and Nanodevices (CRANN) \& Advanced Materials and Bioengineering Research (AMBER) Centre, Trinity College Dublin, Dublin 2, Ireland. Correspondence and requests for materials should be addressed to H.G.M. (email: manninh@tcd.ie)
} 


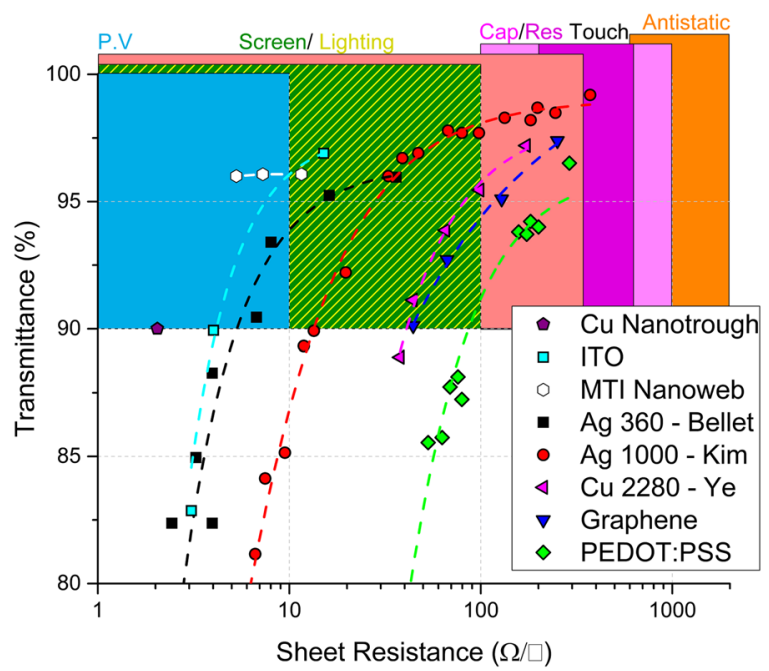

Figure 1. Sheet resistance $\left(R_{\mathrm{s}}\right)$ requirements for various flexible transparent conductor (TC) applications, photovoltaic (PV), screen and lighting, capacitive and resistive touch screens and antistatic coatings (top). Transmittance, $T-R_{\mathrm{s}}$ graph, groups of data points compare the best performing (TC) technologies currently available. Cu Nanotrough \& ITO ${ }^{56}$, MTI Nanoweb ${ }^{57}, \mathrm{Ag}$ nanowires (NWs) with an aspect ratio (AR) of $360^{13}$, $1000^{58}$, Cu NWs with an AR of $2280^{9}$, PEDOT:PSS TC ${ }^{2}$, calculated and experimental graphene $\mathrm{TCs}^{58,59}$.

Modification of the PVP surface layer can give resistive switching memory effects ${ }^{24,25}$, or enhance the thermal and chemical stability of the $\mathrm{Ag} \mathrm{NWNs}^{17,26,27}$.

PVP is necessary during the synthesis process and stabilises the nanowires in solution ${ }^{28}$. Optimisation of the $R_{\mathrm{jxn}}$ value in NWNs typically involves post-processing techniques such as thermal treatment ${ }^{29}$, mechanical pressing $^{30}$, cold welding ${ }^{31}$, optically induced welding ${ }^{32}$, electrochemical Ostwald ripening ${ }^{33}$ or electrical stressing $^{34}$, each of which can dramatically lower the $R_{\mathrm{s}}$. However, in the absence of an electro-optical predictive tool, the effectiveness of these processing methods has been difficult to assess, compare and prescribe for specific applications.

We previously introduced a computational approach to describe the conduction properties of metallic NWNs using a multi-nodal representation (MNR) model which calculates the $R_{\mathrm{s}}$ considering the contributions associated with NW junctions $\left(R_{\mathrm{ixn}}\right)$ and the NW segments (with inner resistances given by $R_{\mathrm{in}}$ ) between them ${ }^{23}$. One of the computational implementations for this model is available in the Supporting Information. Incorporating the inner-wire resistance (which depends on the NW material and diameter) is important but often overlooked. It allows the skeletal resistance of the network (in the limit when $R_{\mathrm{jxn}} \rightarrow 0$ ) to be determined, for which the resulting network is comprised of ballistic NW junctions, representing the ultimate conductivity of the $\mathrm{NWN}^{23}$. It is well known that increasing the NW length to diameter aspect ratio (AR) of the NWs results in lower $R_{\mathrm{s}}$ values and a larger $T^{35,36}$. Thus far, electro-optical models of Ag NWNs have used empirical expressions to describe how $T$ depends on $R_{\mathrm{s}}$ (which itself depends explicitly on material resistivity $(\rho), R_{\mathrm{in}}, R_{\mathrm{jxn}}$ and $\mathrm{AR}$ ); approaches that typically require fitting parameters ${ }^{35,37,38}$, or using other empirically sourced quantities to achieve agreement between experimental and simulated data ${ }^{13,20,39,40}$. The $T-R_{\mathrm{s}}$ curve has a characteristic shape, which was highlighted by Mutiso et al. ${ }^{38}$ who fit an empirical expression with good agreement to a percolative model derived from thin-films ${ }^{40}$. However, none of these semi-empirical approaches are predictive, nor can they accurately describe a wide range of NWNs.

In this work we use the Mie light scattering theory (MLST) of NWNs described by Khanarian et al. to predict the transmittance as a function of the diameter of the NWs and the surface fraction coverage ${ }^{41}$. MLST is an exact theory which has no fitting parameters and is only dependent on the wavelength of incident light, the NW diameter, and the optical constants of the NW material. We build upon the electrical MNR model by incorporating a first principles approach based on MLST of NWs to determine the electro-optical performance of the NWN. This fully predictive model establishes the limits of Ag NWN performance and faithfully captures the behaviour of experimental data from the literature over a wide range of NW ARs. Importantly, this tool can be used to engineer NW systems for different applications in a true materials-by-design approach, allowing an effective comparison of different NWN processing methods that will facilitate the adoption of NWN films in current and next-generation devices.

\section{Results}

The electrical performance of percolating NWNs depends significantly on the properties of the constituent NWs. Physical properties such as length and diameter determine the ultimate conductance potential of the network. The electrical performance of a Ag NWN was simulated using the MNR model for a given AR and wire density, setting $\rho=22.6 \mathrm{n} \Omega \mathrm{m}^{23}$, and $R_{\mathrm{jxn}}=11 \Omega$ corresponding to the median value of the experimentally optimised distribution of junction resistances in Ag/PVP systems (see Fig. 2c) ${ }^{42}$. For the purposes of simulation, the NWs were considered as rigid rods. Singular values of NW length and diameter were used in all computations, although the 

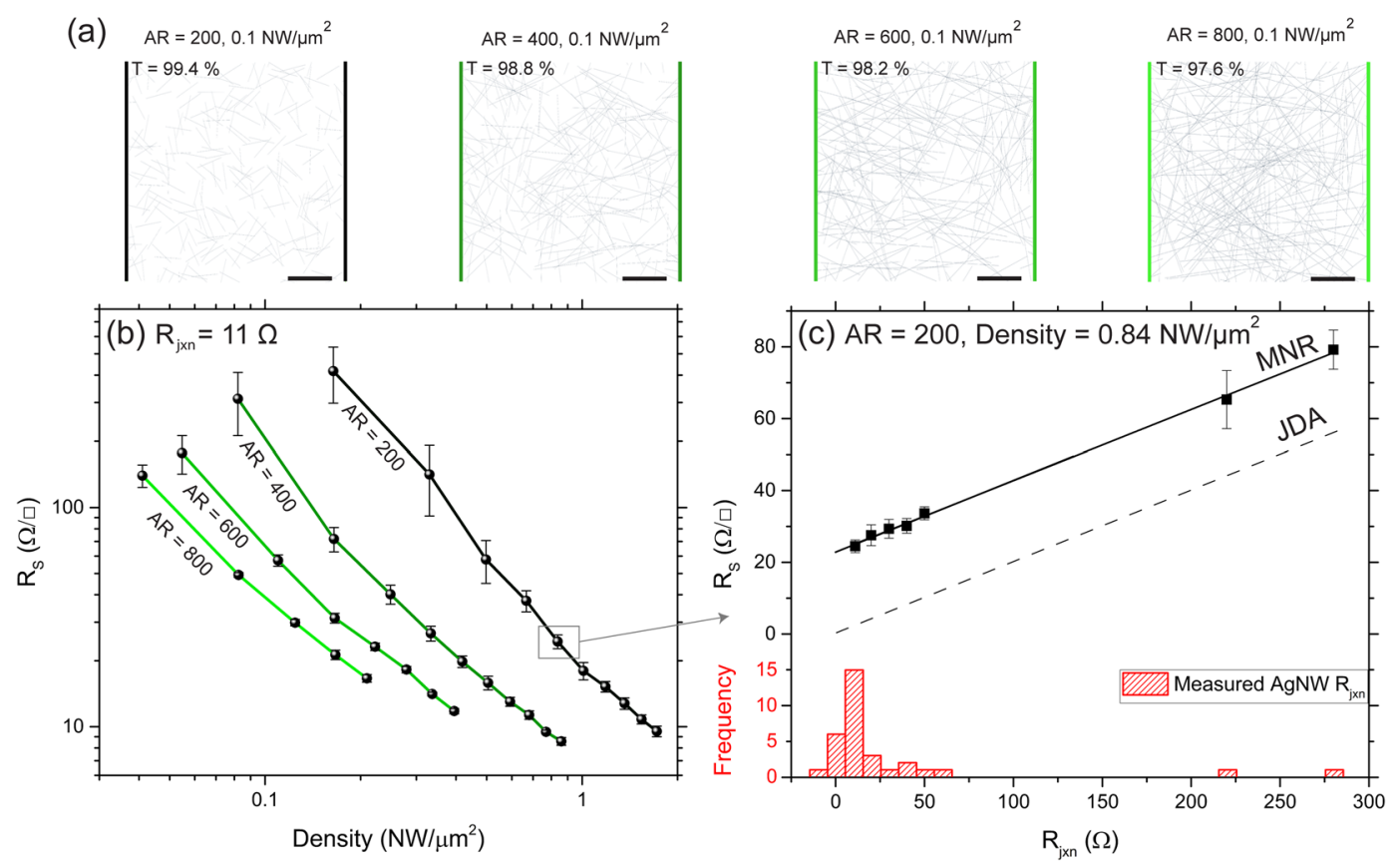

Figure 2. (a) Representative plots of simulated wire networks at a density of $0.1 \mathrm{NWs} / \mu \mathrm{m}^{2}$ for aspect ratios (ARs) of 200, 400, 600 and 800 with nanowire (NW) diameter of $30 \mathrm{~nm}$ and a simulated cell size of $50 \times 50 \mu \mathrm{m}$; the respective transmittance $(T)$ values are shown on each panel. (b) Average sheet resistance $\left(R_{\mathrm{s}}\right)$ as a function of the Ag nanowire network density for four AR values where the NW-NW junction resistance $\left(R_{\text {ixn }}\right)$ is $11 \Omega$ and the NW material resistance is included using the multi-nodal representation (MNR) model. The Ag NW diameter was fixed at $30 \mathrm{~nm}$ for all simulations, the error bars arise from the standard deviation of 10 simulated networks, the simulated cell size for the above ARs was 15, 30, 45 and $55 \mu \mathrm{m}$, respectively. (c) For the highlighted density of $0.84 \mathrm{NW} / \mu \mathrm{m}^{2}$ with $\mathrm{AR}=200 \mathrm{in}(\mathbf{b})$, the average $R_{\mathrm{s}}$ was calculated as a function of the $R_{\mathrm{jxn}}$ using the MNR model, and when the inner resistance is neglected from the calculations in the junction dominated approach (JDA). Below the main plot is a histogram of the experimentally measured $R_{\mathrm{jxn}}$ distribution of $\mathrm{Ag}$ NWs from Bellew et al. ${ }^{42}$.

MNR model is also able to account for more realistic aspects of networks, e.g. dispersion in physical parameters such as length, diameter, $R_{\mathrm{jxn}}$ and the presence of "outlier" junctions. Here, our goal is to avoid unnecessary complexity and show the raw capabilities of the model in describing and predicting real world performance without any fitting parameters; the flexibility of the model enables the incorporation of dispersion and other disorder elements in a straightforward fashion.

Figure 2(a) shows plots of the simulated Ag NWNs at a density of $0.1 \mathrm{NW} / \mu \mathrm{m}^{2}$ for ARs of 200, 400, 600 and 800 ; which corresponds to $T$ values of $99.4 \%, 98.8 \%, 98.2 \%$ and $97.6 \%$, respectively. The diameter of the NWs was fixed at $30 \mathrm{~nm}$ and the simulation box was set at $50 \times 50 \mu \mathrm{m}$ in each case. In Fig. 2(b), the power law dependence of the $R_{\mathrm{s}}$ on NW density is clear for all AR values plotted, consistent with percolative and closed form models of electrical transport within $\mathrm{NWNs}^{22,38,43}$. The importance of including the material resistance in the calculation of the $R_{\mathrm{s}}$ is highlighted in Fig. 2(c), where $R_{\mathrm{s}}$ is calculated as a function of the $R_{\mathrm{jxn}}$ for a junction dominated approach (JDA, dashed black line) NWs have no internal resistance. The MNR model which accounts for the resistance of the wire segments between junctions is shown as a solid black line. When $R_{\mathrm{jxn}} \rightarrow 0$, the MNR model provides the ultimate limit of the network $R_{\mathrm{s}}$, which for an AR of 200 with a density of $0.84 \mathrm{NW} / \mu \mathrm{m}^{2}(T=95 \%)$ is $\sim 20 \Omega / \square$. The distribution on the lower half of the panel represents the range of experimentally measured $R_{\mathrm{jxn}}$ values for $\mathrm{Ag} \mathrm{NWs}{ }^{42}$. As the $R_{\mathrm{jxn}}$ increases, the $R_{\mathrm{s}}$ increases linearly, therefore NWNs must be subjected to processing steps after network formation to reduce the contact resistance between the NWs. These results highlight the importance of $R_{\mathrm{jxn}}$ optimisation in high performance NWNs, moreover, it shows the significant contribution of the $R_{\mathrm{in}}$ to the overall $R_{\mathrm{s}}$, and that the true upper bounds of electrical performance can only be determined when the inner wire resistance is considered.

To estimate the $T$ of a NWN, we begin by considering the extinction coefficient at normal incidence, $C_{\text {ext }}$, which represents the amount of light scattered and absorbed by a single NW from Mie theor $\mathrm{y}^{44}$. The area fraction (AF) describes the projection of the NWs per unit area of the substrate which is defined as the density, $N$, per unit area multiplied by the length $(L)$ and diameter $(D)$ of the NWs.

$$
A F=N \times L \times D
$$

When the thickness of the network is comparable to the diameter of the NWs, the transmittance, $T$, as derived by Khanarian et al. can be expressed as ${ }^{41}$, 


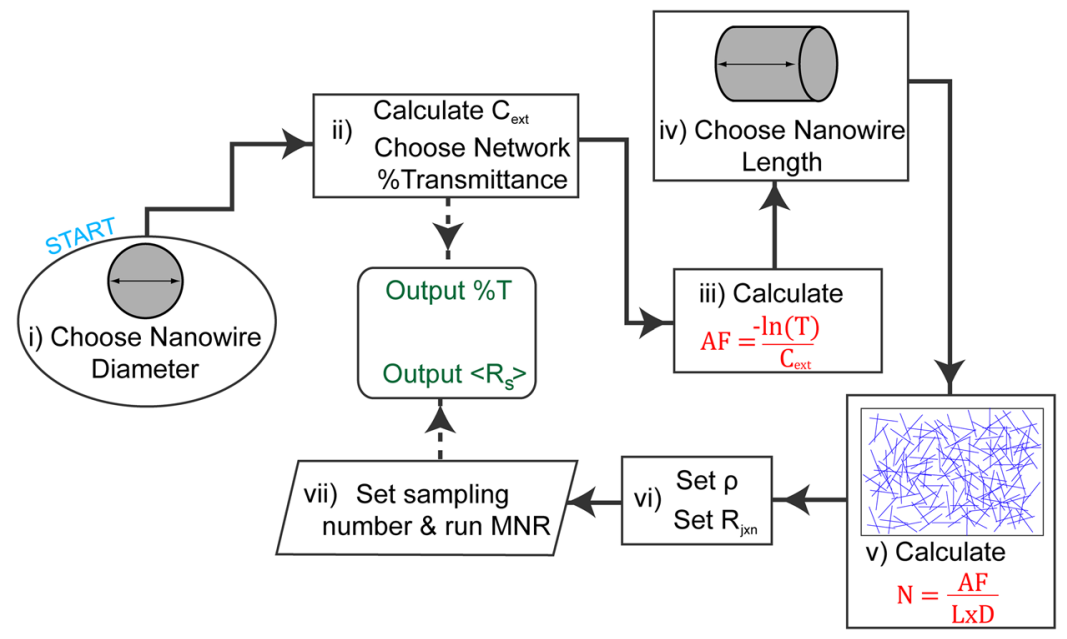

Figure 3. The flow diagram of the multi-nodal representation (MNR) and Mie light scattering theory (MLST) models for the electro-optical behaviours of nanowire networks (NWNs). (i) The diameter of the nanowire (NW) is selected; this is a critical parameter used in step (ii) to determine the extinction coefficient $C_{\text {ext }}$. The desired optical transmittance (T) value of the NWN sample is also set here. (iii) Equation 3 is solved for the area fraction (AF) and (iv) the aspect ratio (AR) of the NWs is set by the NW length. (v) Equation 2 is solved for network density, $N$, which provides the NW density for the randomly generated network samples used in the MNR code. In (vi) the electrical properties, $\rho$, and the interwire junction resistance parameter $\left(R_{\mathrm{jxn}}\right)$ is set. (vii) The MNR algorithm is executed and outputs an average sheet resistance $<R_{\mathrm{s}}>$ for the prescribed number of network samples.

$$
T=e^{-A F \times C_{e x t}}
$$

The flow diagram in Fig. 3 describes the implementation of the combined MNR and MLST models to simulate the electro-optical properties of NWNs. The only necessary inputs to the model are the NW diameter and length. The model then calculates the corresponding network density and determines the $R_{\mathrm{s}}$ given the values of $\rho$ and $R_{\mathrm{jxn}}$. Thus the model not only predicts the performance of a particular network but given experimental $T-R_{\mathrm{s}}$ data for a network of known $D$ and $L$ values it can predict the average $R_{\mathrm{jxn}}$. The only way to alter the $R_{\mathrm{s}}$ for such a network (at a specific value of $T$ ) is to vary the $R_{\mathrm{jxn}}$ value used in the MNR model, which, as previously discussed, can be influenced by different processing techniques. A discussion and analysis of previously reported, empirically derived, electro-optical models is presented in the Supplementary Information as Figs S1 and S2. This data shows that the MNR MLST model describes the expected shape of not only experimentally obtained $T-R_{\mathrm{s}}$ data, but can accurately describe the synthetic data generated from previously reported semi-empirical models.

Figure 4(a-d) shows the MNR calculated plots of the $T-R_{\mathrm{s}}$ for Ag NWNs with various AR values and for mean $R_{\mathrm{jxn}}$ values of $11 \Omega, 100 \Omega, 1000 \Omega$ and in the case of perfect junctions with a resistance of $0 \Omega$. Each point in Fig. 4 was calculated by following the process outlined in Fig. 3 where $\rho$ is fixed, and $R_{\mathrm{jxn}}$ is varied. Figure 4(a) highlights the importance of optimising $R_{\mathrm{ixn}}$ in the technologically relevant $T$ region. It is important to note that when AR $>200$ (Fig. 4(a,b)) at a $T=90 \%$, the simulations predict that the $R_{\mathrm{s}}$ will be $<100 \Omega / \square$, which is acceptable for touch screen applications, however, solar cells and OLED electrodes require much lower $R_{\mathrm{s}}(\sim 10 \Omega / \square)$ which can only be achieved by $R_{\mathrm{jxn}}$ optimisation $\left(R_{\mathrm{jxn}}<100 \Omega\right)$ of highly transparent networks $(T>95 \%)$. The truncation of the simulation in Fig. $4(\mathrm{c}, \mathrm{d})$ is due to the prohibitive computational requirements to calculate sufficiently dense networks with $T<93 \%$ for $\mathrm{AR}=600$ and $T<95 \%$ for $\mathrm{AR}=800$ samples. The linear dependence of $T$ with respect to the network density is plotted in Fig. S3, a dependence that is experimentally observed and has been theoretically derived by Ainsworth et al. ${ }^{39}$.

In a physical NWN sample, it is impossible to probe individual $R_{\mathrm{jxn}}$ values via experimental means. A strength of the MNR model is that it provides insights into the average contribution of the $R_{\mathrm{jxn}}$ to the measured $R_{\mathrm{s}}$. By combining MNR and MLST models, we can begin to benchmark experimental data developing a materials-by-design approach to NWN-based TCs. Setting a theoretical benchmark for NWN systems allows a better comparison of synthesis methods, deposition procedures and specific post-processing techniques. For example, thermal annealing can hugely improve the $R_{\mathrm{s}}$ of as-deposited networks, but the anneal temperature and time must be chosen carefully and will depend on NW diameter and the thermal properties of the substrate. Figure 5(a) shows the improvement in the $R_{\mathrm{s}}$ by post-deposition annealing. Madaria et al ${ }^{45}$ measured the performance of Ag NWs with $\mathrm{AR}=166$ pre and post anneal. The hollow square data points show the $R_{\mathrm{s}}$ before annealing the NWN films. The black squares show the marked decrease of the $R_{\mathrm{s}}$ after an anneal time of $20 \mathrm{~min}$ at $200^{\circ} \mathrm{C}$. The authors report the length and diameter values of the constituent NWs, which determine the densities of these networks (from Eq. 3) for the simulations. The MNR model can compute the electrical performance of these networks separating the $R_{\mathrm{s}}$ into the contributions of the wire material and the $R_{\mathrm{jxn}}$, the latter being varied to obtain a good fit to the experimental data. The dotted blue curve shows the average $R_{\mathrm{s}}$ results that match the as-deposited NWNs with $R_{\mathrm{jxn}}=60 \Omega$. The width of the shaded coloured area represents both the horizontal component of the error from 

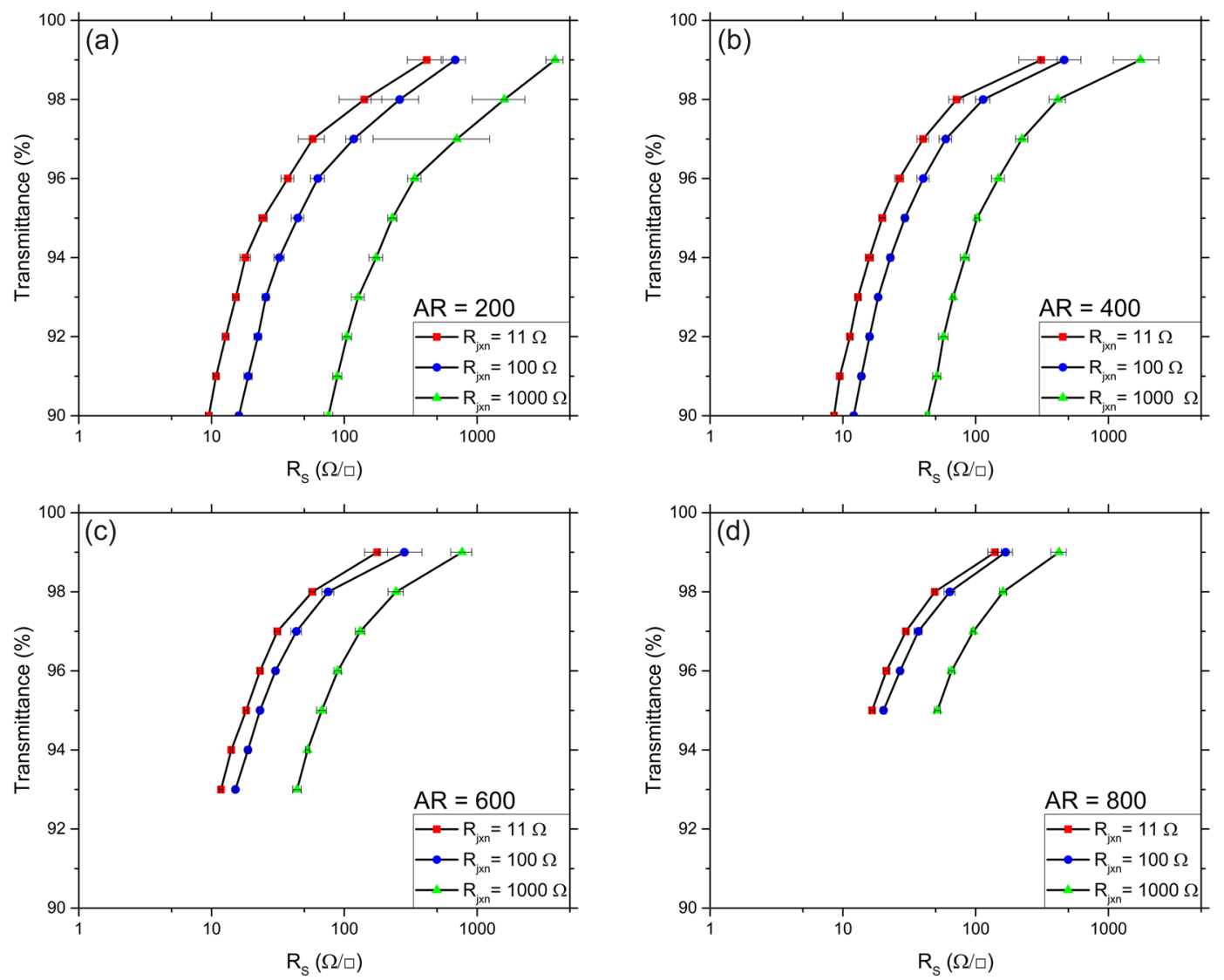

Figure 4. (a-d) Calculated sheet resistance $\left(R_{\mathrm{s}}\right)$ for four aspect ratios (ARs) using the multi-nodal representation (MNR) and Mie light scattering theory (MLST) models for junction resistance $\left(R_{\mathrm{jxn}}\right)$ values of 11,100 and $1000 \Omega$. Panels $(\mathbf{c}, \mathbf{d})$ have less points due to machine memory limitations. The error bars arise from the standard deviation of $10 \mathrm{Ag}$ nanowire networks (NWNs) for each data point. The transmittance is calculated using Eqs 2 and 3 of the Mie theory method which is explained in the main text.

the standard deviation of the $R_{\mathrm{s}}$ for 10 simulated networks, and the vertical spread in $T$ due to the uncertainty in the NW diameter value when calculating $C_{\text {ext }}$. After the annealing step, the $T-R_{\mathrm{s}}$ curve is better described by $R_{\mathrm{ixn}}=11 \Omega$, suggesting the annealing treatment has produced highly optimised TC films with extremely low $R_{\mathrm{s}}$ for that particular AR. However, even at this optimised $R_{\mathrm{jxn}}$ value, the NWNs fail the $R_{\mathrm{s}}$ requirement for photovoltaic applications and barely reaches the requirements for screen/lighting technologies (see Fig. 1).

Another example of NWN post processing benchmarking is shown in Fig. 5(b) using the data from Liu et al. ${ }^{31}$. In their study, moisture-induced capillary-forces were shown to cause a self-limiting cold welding of the NW junctions, hence reducing the $R_{\mathrm{s}}$. The hollow square data points show the $T-R_{\mathrm{s}}$ data of the as-prepared samples. The effect of the moisture treatment significantly reduces the $R_{\mathrm{s}}$ of the Ag NWNs and causes the network to adopt the "expected shape" curve which is an important indicator of the performance of the network as predicted by the MNR MLST models (cf. Fig. 4). We can apply the MNR MLST models to determine the $R_{\mathrm{ixn}}$ value needed to describe the network (comprised of NWs with $\mathrm{AR}=222$ ). The resulting blue shaded curve for the moisture treated samples has the expected $T-R_{\mathrm{s}}$ shape, and is well described by $R_{\mathrm{jxn}}=750 \Omega$. The failure of the as-prepared film to exhibit the same shape as the moisture treated samples suggest that the network connectivity is poorly established or that there is a significant spread in $R_{\mathrm{jxn}}$ values. The power of MNR MLST is that it can determine the $R_{\mathrm{jxn}}$ values necessary to describe the measured $R_{\mathrm{s}}$ values in the as-prepared films - the spread is between 20 $\mathrm{k} \Omega$ and $40 \mathrm{k} \Omega$ allowing a rapid evaluation of processing techniques used to form the network, which hitherto was not possible.

While it is obvious that moisture treatment has made a significant improvement to the measured $R_{s}$, it has not produced the most optimised NWN yet. MNR MLST can predict the effect of additional optimisation. By decreasing the $R_{\mathrm{jxn}}$ to a value of $11 \Omega$ (red shaded curve, Fig. 5(b)), the simulations suggest further room for improvement. In-situ resistance measurements during thermal annealing for NWNs of a similar diameter suggest that an annealing temperature of $200{ }^{\circ} \mathrm{C}$ is required to realise the most conducting $\mathrm{NWN}$ films ${ }^{46}$. Analysis of additional systems with $\mathrm{AR}=182,306,440,600,641,760,800,1000$ and 2000 are shown in section 2 of the Supplementary Information confirming that our predictive model provides good agreement with experimental data. In some rare cases, MLST over-estimates the optical transmittance and, in some cases, MNR underestimates the $R_{\mathrm{s}}$. A discussion of deviations from the model are included in sections 3 and 4 of the Supplementary Information. Other factors that can affect the electro-optical predictions of our model include the inherent 

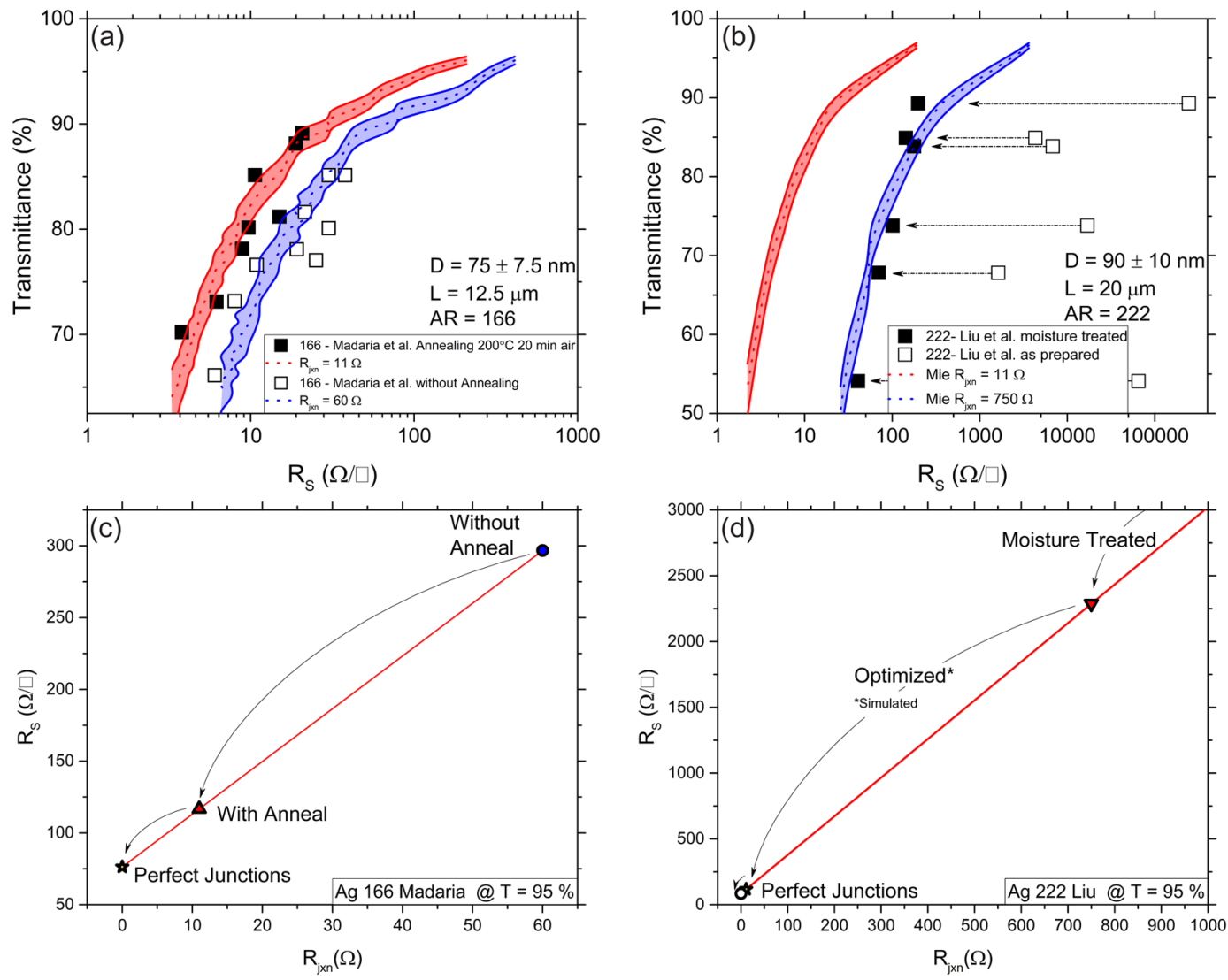

Figure 5. (a,b) The square data points on each panel represent experimental data of Ag nanowire networks (NWNs) taken from reported literature values with various aspect ratios (ARs) and post-deposition processing methods. The hollow data points in (a) show Ag NWNs which were measured after being deposited, while the black squares show data for NWN samples which were annealed for 20 minutes at $200^{\circ} \mathrm{C}$. In (b), the hollow squares show the electro-optical performance of as-prepared Ag NWNs with the black squares showing an increase in performance after the same NWNs were moisture treated. The dashed colored lines show the average sheet resistance $\left(R_{\mathrm{s}}\right)$ predictions of the performance of the wire systems simulated using the multinodal representation (MNR) model where, in red, the junction resistance $\left(R_{\mathrm{jxn}}\right)$ is set to $11 \Omega$ and in blue the $R_{\mathrm{jxn}}$ was increased to (a) $60 \Omega$, and (b) $750 \Omega$ to fit the experimentally reported data. The red and blue shaded areas bounded by the continuous line represents the standard deviation of the $R_{\mathrm{s}}$ of 10 simulated samples, and the error associated in the transmittance $(T)$ calculation from Mie theory using the reported spread of diameter values. (c,d) $R_{\mathrm{s}}$ vs $R_{\mathrm{jxn}}$ analysis performed at $T=95 \%$ for the NWN in (a,b), respectively. In (c), a significant decrease in the $R_{\mathrm{s}}$ is observed when the NWNs are annealed, and in (d) the as-deposited network (data point not shown) is estimated to have $R_{\mathrm{s}}=100 \mathrm{k} \Omega$ which yields by linear extrapolation $R_{\mathrm{jxn}}$ values of $34 \mathrm{k} \Omega$. Moisture treated NWNs have a much lower $R_{\mathrm{s}}$, but simulations suggest this could be further optimised as $R_{\mathrm{jxn}}=11 \Omega$. The ultimate limit of the $R_{\mathrm{s}}$ can be extrapolated by considering $R_{\mathrm{jxn}} \rightarrow 0$ where only the material resistance limits the electrical performance of the network.

flexibility of the NWs and the diameter dependent persistence length which is known to play an important role in the connectivity of the networks ${ }^{47,48}$. At this point, for the sake of simplicity, our simulations only account for rigid rods and fixed persistence lengths. Nonetheless, the current model is flexible and can be extended to incorporate these elements.

Network optimisation can be graphically described by the combined MNR and MLST model. In Fig. 5(c,d), the $R_{\mathrm{s}}$ is calculated as a function of the $R_{\mathrm{jxn}}$ for the data presented in Fig. 5(a,b) at $T=95 \%$. This linear relationship has been previously reported by our group when first implementing the MNR model and now serves as a roadmap for predicting the ultimate performance of NWN materials ${ }^{23}$. The linear decrease in the $R_{\mathrm{s}}$ assumes a decrease in the resistance of all junctions in the network, however in reality, some paths within the network may not be conducting initially and may require one of the processing steps previously discussed. As these additional paths become conducting, the simple linear relationship shown here may be curved or stepped ${ }^{49,50}$. The two datasets in Fig. 5(a,b) (two further examples for AR 306 and 760 are presented in Fig. S18) initially had $R_{\mathrm{jxn}}$ which were predicted to be higher than the optimised value. The ability of the model to separately consider junction and inner-wire resistances allows for the ultimate performance of the NWN to be determined, which occurs when $R_{\mathrm{jxn}} \rightarrow 0 \Omega$. This allows an estimate of how close a NWN film is to having perfect interwire contacts, and enables a rigorous and quantitative analysis of post-processing techniques. While the tunability of the $R_{\mathrm{s}}$ in metallic NWNs 
via a combination of AR, NW density, material choice and $R_{\mathrm{jxn}}$ makes these materials attractive to numerous applications, the presence of a $R_{\mathrm{jxn}}$ between wires will always limit performance. Perfect lossless junctions may not be achievable in solution deposited NWNs but are a feature of seamless junction networks such as crackled template networks ${ }^{51,52}$.

\section{Conclusions}

In this work, we combined two computational methods to deliver the first fully predictive model of both the electrical and optical performances of metal nanowire networks. The multi-nodal representation (MNR) model which calculates the sheet resistance $\left(R_{\mathrm{s}}\right)$ of the nanowire networks (NWNs) considers both the resistance contribution of the nanowire segments and the nanowire (NW) junctions. Using experimentally measured resistivity and junction resistance values for the case of Ag NWNs, we show how the $R_{\mathrm{s}}$ depends on the nanowire length/ diameter aspect ratio (AR). The inner-wire resistance is also important as it determines the lowest attainable $R_{\mathrm{s}}$, and the inclusion of the skeletal resistance allows the magnitude of the junction resistance between overlapping wires, $R_{\mathrm{jxn}}$, to reveal the level of optimisation of the NWN through post-processing steps. The Mie light scattering theory (MLST) model describes the optical transmittance of NWNs according to its fundamental physical properties and network density. This simple but robust model achieves excellent agreement with experimental data over a wide range of NW ARs. The results of this work show that simulation of NWNs is an important tool in benchmarking the efficacies of post-processing methods, and offers a strategic approach to exploring the potential applications of NWN materials and guiding the synthesis of systems for specific needs. NWNs are well suited as replacements for ITO (tin-doped indium oxide) in a wide variety of current and emerging flexible devices. The development of a predictive model for these materials is an important step towards a materials-by-design approach for transparent conductor applications.

\section{Methods}

MNR simulations were implemented in the Python language, the code for which is available in the Supplementary Information. The networks were generated according to the input parameters of wire diameter, wire length, wire density (number of wires per unit area) and simulation box length, which defines the squared area of the box where wires are randomly placed. The simulation box was always set larger than two times the wire length. Ag NW material resistivity is $\rho=22.6 \mathrm{n} \Omega \mathrm{m}$, and the $R_{\mathrm{ixn}}$ was varied according to the experiment. The MNR voltage grid scheme, which is described in detail in reference ${ }^{23}$ of the manuscript maps the spatial coordinates of the interwire connection points, and assigns either an interwire junction resistance $R_{\mathrm{ixn}}$, or an inner-wire segment with a resistance calculated by $R_{\text {in }}=\rho l / A$, where $l$ is the length of the segment and $A$ is the cross-sectional area of the wire. The corresponding resistance matrix is solved using Kirchhoff's circuit law to obtain the $R_{\mathrm{s}}$ of the sample. The number of representative NWN samples of the ensemble was set to 10 . The program output the average $R_{s}$, and the standard deviation of the $R_{s}$.

The $C_{\text {ext }}$ is calculated using the MatScat ${ }^{53}$ (Mie theory for infinite cylinders) implementation by Schäfer et al. ${ }^{54}$ and depends only on the NW diameter and the optical constants for the metal. The refractive index $(n)$ and extinction coefficient $(k)$ used for $\mathrm{Ag}$ is, $n=0.13936, k=3.5604$ at $\lambda=546 \mathrm{~nm}^{55}$. From Equations 2 and 3 , the NWN density corresponds to a $T$ value. $T-R_{\mathrm{s}}$ data of NWNs across a wide variety of aspect ratios was gathered from 17 publications, where the NW lengths and diameters were reported. The $T$ values reported by these publications were converted into NWN densities which were calculated by MNR through the process outlined in Fig. 3. Where a spread in diameter values was reported, the upper and lower bounds described a variation in $C_{\text {ext }}$ and hence $T$ which is displayed as the shaded areas on the $T-R_{\mathrm{s}}$ graphs.

\section{Data Availability}

All data generated or analysed during this study are included in this published article (and its Supplementary Information Files), the datasets are also available from the corresponding author on reasonable request.

\section{References}

1. Langley, D. et al. Flexible transparent conductive materials based on silver nanowire networks: a review. Nanotechnology 24, 452001, https://doi.org/10.1088/0957-4484/24/45/452001 (2013).

2. Elschner, A. \& Lovenich, W. Solution-deposited PEDOT for transparent conductive applications. Mrs Bulletin 36, 794-798, https:// doi.org/10.1557/mrs.2011.232 (2011).

3. Scardaci, V., Coull, R. \& Coleman, J. N. Very thin transparent, conductive carbon nanotube films on flexible substrates. Applied Physics Letters 97, 023114-023114-023113, https://doi.org/10.1063/1.3462317 (2010).

4. Bae, S., Kim, S. J., Shin, D., Ahn, J. H. \& Hong, B. H. Towards industrial applications of graphene electrodes. Physica Scripta T146, 014024, https://doi.org/10.1088/0031-8949/2012/T146/014024(2012).

5. Hecht, D. S., Hu, L. \& Irvin, G. Emerging transparent electrodes based on thin films of carbon nanotubes, graphene, and metallic nanostructures. Adv Mater 23, 1482-1513, https://doi.org/10.1002/adma.201003188 (2011).

6. Kiruthika, S., Rao, K. D. M., Kumar, A., Gupta, R. \& Kulkarni, G. U. Metal wire network based transparent conducting electrodes fabricated using interconnected crackled layer as template. Materials Research Express 1, 026301, https://doi.org/10.1088/2053$1591 / 1 / 2 / 026301(2014)$.

7. Liu, C. H. \& Yu, X. Silver nanowire-based transparent, flexible, and conductive thin film. Nanoscale Res Lett 6, 75, https://doi. org/10.1186/1556-276X-6-75 (2011).

8. Lyons, P. E. et al. High-Performance Transparent Conductors from Networks of Gold Nanowires. J Phys Chem Lett 2, 3058-3062, https://doi.org/10.1021/jz201401e (2011).

9. Ye, S. et al. A rapid synthesis of high aspect ratio copper nanowires for high-performance transparent conducting films. Chem Commun 50, 2562-2564, https://doi.org/10.1039/c3cc48561g (2014).

10. Scardaci, V., Coull, R., Lyons, P. E., Rickard, D. \& Coleman, J. N. Spray deposition of highly transparent, low-resistance networks of silver nanowires over large areas. Small 7, 2621-2628, https://doi.org/10.1002/smll.201100647 (2011).

11. Moon, H., Won, P., Lee, J. \& Ko, S. H. Low-haze, annealing-free, very long Ag nanowire synthesis and its application in a flexible transparent touch panel. Nanotechnology 27, 295201, https://doi.org/10.1088/0957-4484/27/29/295201 (2016). 
12. Kim, D. J. et al. Roll-to-roll slot-die coating of $400 \mathrm{~mm}$ wide, flexible, transparent Ag nanowire films for flexible touch screen panels. Sci Rep-Uk 6, 34322, https://doi.org/10.1038/srep34322 (2016).

13. Bellet, D. et al. Transparent Electrodes Based on Silver Nanowire Networks: From Physical Considerations towards Device Integration. Materials (Basel) 10, 570, https://doi.org/10.3390/ma10060570 (2017).

14. Yang, L. et al. Solution-processed flexible polymer solar cells with silver nanowire electrodes. ACS Appl Mater Interfaces 3, 4075-4084, https://doi.org/10.1021/am2009585 (2011).

15. Cann, M. et al. High performance transparent multi-touch sensors based on silver nanowires. Materials Today Communications 7 , 42-50, https://doi.org/10.1016/j.mtcomm.2016.03.005 (2016).

16. Park, H. G. et al. Silver Nanowire Networks as Transparent Conducting Films for Liquid Crystal Displays. Ecs Solid State Letters 4, R50-R52, https://doi.org/10.1149/2.0031510ssl (2015).

17. Lagrange, M. et al. Understanding the mechanisms leading to failure in metallic nanowire-based transparent heaters, and solution for stability enhancement. Nanotechnology 28, 055709, https://doi.org/10.1088/1361-6528/28/5/055709 (2017).

18. Wu, C., Kim, T. W., Li, F. \& Guo, T. Wearable Electricity Generators Fabricated Utilizing Transparent Electronic Textiles Based on Polyester/Ag Nanowires/Graphene Core-Shell Nanocomposites. ACS Nano 10, 6449-6457, https://doi.org/10.1021/ acsnano.5b08137 (2016).

19. Moon, H. et al. Ag/Au/Polypyrrole Core-shell Nanowire Network for Transparent, Stretchable and Flexible Supercapacitor in Wearable Energy Devices. Sci Rep-Uk 7, 41981, https://doi.org/10.1038/srep41981 (2017).

20. De, S. et al. Silver Nanowire Networks as Flexible, Transparent, Conducting Films: Extremely High DC to Optical Conductivity Ratios. ACS Nano 3, 1767-1774, https://doi.org/10.1021/nn900348c (2009).

21. Benoy, M. D., Mohammed, E. M., Suresh, B. M., Binu, P. J. \& Pradeep, B. Thickness dependence of the properties of indium tin oxide (ITO) films prepared by activated reactive evaporation. Brazilian Journal of Physics 39, 629-632, https://doi.org/10.1590/S010397332009000600003 (2009)

22. O'Callaghan, C., Rocha, C. G., Manning, H. G., Boland, J. J. \& Ferreira, M. S. Effective medium theory for the conductivity of disordered metallic nanowire networks. Phys Chem Chem Phys 18, 27564-27571, https://doi.org/10.1039/c6cp05187a (2016).

23. Rocha, C. G. et al. Ultimate conductivity performance in metallic nanowire networks. Nanoscale 7, 13011-13016, https://doi. org/10.1039/c5nr03905c (2015).

24. Manning, H. G., Biswas, S., Holmes, J. D. \& Boland, J. J. Nonpolar Resistive Switching in Ag@TiO2 Core-Shell Nanowires. ACS Appl Mater Interfaces 9, 38959-38966, https://doi.org/10.1021/acsami.7b10666 (2017).

25. Du, H. et al. Engineering Silver Nanowire Networks: From Transparent Electrodes to Resistive Switching Devices. ACS Appl Mater Interfaces 9, 20762-20770, https://doi.org/10.1021/acsami.7b04839 (2017).

26. Hwang, B. et al. Highly Flexible and Transparent Ag Nanowire Electrode Encapsulated with Ultra-Thin $\mathrm{Al}_{2} \mathrm{O}_{3}$ : Thermal, Ambient, and Mechanical Stabilities. Sci Rep-Uk 7, 41336, https://doi.org/10.1038/srep41336 (2017).

27. Lee, D. G., Lee, D., Yoo, J. S., Lee, S. \& Jung, H. S. Effective passivation of Ag nanowire-based flexible transparent conducting electrode by TiO 2 nanoshell. Nano Convergence 3, 20, https://doi.org/10.1186/s40580-016-0080-z (2016).

28. Coskun, S., Aksoy, B. \& Unalan, H. E. Polyol Synthesis of Silver Nanowires: An Extensive Parametric Study. Crystal Growth \& Design 11, 4963-4969, https://doi.org/10.1021/cg200874g (2011).

29. Langley, D. P. et al. Metallic nanowire networks: effects of thermal annealing on electrical resistance. Nanoscale 6, 13535-13543, https://doi.org/10.1039/c4nr04151h (2014).

30. Tokuno, T. et al. Fabrication of silver nanowire transparent electrodes at room temperature. Nano Research 4, 1215-1222, https://doi. org/10.1007/s12274-011-0172-3 (2011).

31. Liu, Y. et al. Capillary-Force-Induced Cold Welding in Silver-Nanowire-Based Flexible Transparent Electrodes. Nano Lett 17, 1090-1096, https://doi.org/10.1021/acs.nanolett.6b04613 (2017).

32. Ha, J., Lee, B. J., Hwang, D. J. \& Kim, D. Femtosecond laser nanowelding of silver nanowires for transparent conductive electrodes. Rsc Advances 6, 86232-86239, https://doi.org/10.1039/c6ra19608j (2016).

33. Lee, H.-J. et al. Spontaneous and Selective Nano-Welding of Silver Nanowires by Electrochemical Ostwald Ripening and High Electrostatic Potential at the Junctions for High-Performance Stretchable Transparent Electrodes. ACS Appl Mater Interfaces 10, 14124-14131, https://doi.org/10.1021/acsami.8b00837 (2018).

34. Nirmalraj, P. N. et al. Manipulating connectivity and electrical conductivity in metallic nanowire networks. Nano Lett 12, 5966-5971, https://doi.org/10.1021/nl303416h (2012).

35. Large, M. J. et al. Predicting the optoelectronic properties of nanowire films based on control of length polydispersity. Sci Rep-Uk 6, 25365, https://doi.org/10.1038/srep25365 (2016).

36. Sorel, S., Lyons, P. E., De, S., Dickerson, J. C. \& Coleman, J. N. The dependence of the optoelectrical properties of silver nanowire networks on nanowire length and diameter. Nanotechnology 23, 185201, https://doi.org/10.1088/0957-4484/23/18/185201 (2012).

37. Bergin, S. M. et al. The effect of nanowire length and diameter on the properties of transparent, conducting nanowire films. Nanoscale 4, 1996-2004, https://doi.org/10.1039/c2nr30126a (2012).

38. Mutiso, R. M., Sherrott, M. C., Rathmell, A. R., Wiley, B. J. \& Winey, K. I. Integrating simulations and experiments to predict sheet resistance and optical transmittance in nanowire films for transparent conductors. ACS Nano 7, 7654-7663, https://doi.org/10.1021/ nn403324t (2013)

39. Ainsworth, C. A., Derby, B. \& Sampson, W. W. Interdependence of Resistance and Optical Transmission in Conductive Nanowire Networks. Advanced Theory and Simulations 1, 1700011, https://doi.org/10.1002/adts.201700011 (2018).

40. De, S., King, P. J., Lyons, P. E., Khan, U. \& Coleman, J. N. Size effects and the problem with percolation in nanostructured transparent conductors. ACS Nano 4, 7064-7072, https://doi.org/10.1021/nn1025803 (2010).

41. Khanarian, G. et al. The optical and electrical properties of silver nanowire mesh films. Journal of Applied Physics 114, 024302, https://doi.org/10.1063/1.4812390 (2013).

42. Bellew, A. T., Manning, H. G., Rocha, C. G., Ferreira, M. S. \& Boland, J. J. Resistance of Single Ag Nanowire Junctions and Their Role in the Conductivity of Nanowire Networks. ACS Nano 9, 11422-11429, https://doi.org/10.1021/acsnano.5b05469 (2015).

43. Forro, C., Demko, L., Weydert, S., Voros, J. \& Tybrandt, K. Predictive Model for the Electrical Transport within Nanowire Networks. ACS Nano 12, 11080-11087, https://doi.org/10.1021/acsnano.8b05406 (2018).

44. Bohren, C. F. \& Huffman, D. R. Absorption and scattering of light by small particles. (John Wiley \& Sons, 2008).

45. Madaria, A. R., Kumar, A., Ishikawa, F. N. \& Zhou, C. W. Uniform, Highly Conductive, and Patterned Transparent Films of a Percolating Silver Nanowire Network on Rigid and Flexible Substrates Using a Dry Transfer Technique. Nano. Research 3, 564-573, https://doi.org/10.1007/s12274-010-0017-5 (2010).

46. Lagrange, M. et al. Optimization of silver nanowire-based transparent electrodes: effects of density, size and thermal annealing. Nanoscale 7, 17410-17423, https://doi.org/10.1039/c5nr04084a (2015).

47. Hicks, J., Li, J. Y., Ying, C. \& Ural, A. Effect of nanowire curviness on the percolation resistivity of transparent, conductive metal nanowire networks. Journal of Applied Physics 123, 204309, https://doi.org/10.1063/1.5029896 (2018).

48. Langley, D. P., Lagrange, M., Nguyen, N. D. \& Bellet, D. Percolation in networks of 1-dimensional objects: comparison between Monte Carlo simulations and experimental observations. Nanoscale Horizons 3, 545-550, https://doi.org/10.1039/c8nh00066b (2018).

49. O'Callaghan, C. et al. Collective capacitive and memristive responses in random nanowire networks: Emergence of critical connectivity pathways. Journal of Applied Physics 124, 152118, https://doi.org/10.1063/1.5037817 (2018). 
50. Manning, H. G. et al. Emergence of winner-takes-all connectivity paths in random nanowire networks. Nat Commun 9, 3219, https://doi.org/10.1038/s41467-018-05517-6 (2018).

51. Rao, K., Gupta, R. \& Kulkarni, G. U. Fabrication of Large Area, High-Performance, Transparent Conducting Electrodes Using a Spontaneously Formed Crackle Network as Template. Advanced Materials Interfaces 1, https://doi.org/10.1002/admi.201400090 (2014).

52. Hossain, M. et al. Transparent, Flexible Silicon Nanostructured Wire Networks with Seamless Junctions for High-Performance Photodetector Applications. ACS Nano 12, 4727-4735, https://doi.org/10.1021/acsnano.8b01387 (2018).

53. Schäfer, J. MatScat Matlab Package, https://www.mathworks.com/matlabcentral/fileexchange/36831-matscat (2012).

54. Schafer, J., Lee, S. C. \& Kienle, A. Calculation of the near fields for the scattering of electromagnetic waves by multiple infinite cylinders at perpendicular incidence. J Quant Spectrosc Ra 113, 2113-2123, https://doi.org/10.1016/j.jqsrt.2012.05.019 (2012).

55. Johnson, P. B. \& Christy, R.-W. Optical constants of the noble metals. Physical Review B 6, 4370, https://doi.org/10.1103/ PhysRevB.6.4370 (1972).

56. He, L. X. \& Tjong, S. C. Nanostructured transparent conductive films: Fabrication, characterization and applications. Mat Sci Eng R 109, 1-101, https://doi.org/10.1016/j.mser.2016.08.002 (2016).

57. Aryal, M. et al. In SID Symposium Digest of Technical Papers. 194-196 (Wiley Online Library).

58. Kim, T. et al. Uniformly interconnected silver-nanowire networks for transparent film heaters. Advanced Functional Materials 23, 1250-1255, https://doi.org/10.1002/adfm.201202013 (2013).

59. Mattevi, C., Kim, H. \& Chhowalla, M. A review of chemical vapour deposition of graphene on copper. Journal of Materials Chemistry 21, 3324-3334, https://doi.org/10.1039/c0jm02126a (2011).

\section{Acknowledgements}

The authors wish to acknowledge funding from the European Research Council (ERC) under Advanced Grant 321160. This publication has emanated from research supported by grants from Science Foundation Ireland (SFI) under P.I Grant 16/IA/4462 and the SFI Research Centre, AMBER under Grant number SFI/12/RC/2278. The Research IT Unit at Trinity College Dublin, the WestGrid (www.westgrid.ca) and Compute Canada Calcul Canada (www.computecanada.ca) are also acknowledged for computational resources.

\section{Author Contributions}

H.G.M. wrote the paper and performed the MNR and MLST simulations. C.G.R. and C.O'.C. developed the MNR computational model and assisted with the MLST simulations. M.S.F. developed the computational model; and J.J.B. led overall effort. All authors discussed and commented on the manuscript and on the results.

\section{Additional Information}

Supplementary information accompanies this paper at https://doi.org/10.1038/s41598-019-47777-2.

Competing Interests: The authors declare no competing interests.

Publisher's note: Springer Nature remains neutral with regard to jurisdictional claims in published maps and institutional affiliations.

(c) (i) Open Access This article is licensed under a Creative Commons Attribution 4.0 International License, which permits use, sharing, adaptation, distribution and reproduction in any medium or format, as long as you give appropriate credit to the original author(s) and the source, provide a link to the Creative Commons license, and indicate if changes were made. The images or other third party material in this article are included in the article's Creative Commons license, unless indicated otherwise in a credit line to the material. If material is not included in the article's Creative Commons license and your intended use is not permitted by statutory regulation or exceeds the permitted use, you will need to obtain permission directly from the copyright holder. To view a copy of this license, visit http://creativecommons.org/licenses/by/4.0/.

(C) The Author(s) 2019 